\title{
Surface micropattern resists bacterial contamination transferred by healthcare practitioners
}

\begin{abstract}
Environmental contamination contributes to an estimated $20-40 \%$ of all hospital-acquired infections (HAI). Infection control practices continue to improve, but multipronged approaches are necessary to fully combat the diversity of nosocomial pathogens and emerging multidrug resistant organisms. The Sharklet ${ }^{\mathrm{TM}}$ micropattern, inspired from the microtopography of shark skin, was recently shown to significantly reduce surface contamination but has not been evaluated in a clinical setting. The focus of this study was the transfer of bacteria onto micropatterned surfaces compared to unpatterned surfaces in a clinical simulation environment involving healthcare practitioners. Physician volunteers were recruited to participate in an emergency medicine scenario involving a contact-precaution patient with an acute pulmonary embolism. Prior to scenario initiation, Staphylococcus aureus was inoculated onto the leg of a simulation mannequin and fresh micropatterned and unpatterned surface films were placed on a code cart, cardiac defibrillator shock button, and epinephrine medication vial. Six physicians interacted with micropatterned surfaces and five physicians interacted with unpatterned surfaces in separate scenarios. Bacterial load loss from the first contact location (control film over the femoral pulse) to subsequent unpatterned or micropatterned surface test locations was quantified as a $\log$ reduction (LR) for each surface type.

The code cart, cardiac defibrillator button, and medication vial locations with micropatterned surfaces resulted in LRs that were larger than the unpatterned LRs by $0.64(p=0.146)$, $1.14(p=0.023)$, and $0.58(p=0.083)$ respectively for each location. The geometric mean $\mathrm{CFU} / \mathrm{RODAC}$ at the first control surface touched at the femoral pulse pads ranged from 175-250 CFU/RODAC (95\% confidence interval). Thus, the micropatterned LRs were consistently greater than the unpatterned LRs, substantiating the micropattern-dependent reduction of microorganism transfer. Principle component analysis showed that the LRs for the code cart and the cardiac defibrillator button highly covaried. Thus, a single mean LR was calculated from these two locations for each surface type; 5.4 times more bacteria attached to the unpatterned surfaces compared to the micropatterned surfaces $(p=0.058)$. The simulated clinical scenario involving healthcare practitioners demonstrated that the micropatterned surface reduced the transfer of bacterial contamination based on the larger LRs for the micropatterned surface compared to control surfaces. Further investigation in hospital rooms where patients are receiving care will ultimately reveal the capability of micropatterned surfaces to minimize the incidence of HAIs.
\end{abstract}

Keywords: contamination, Staphylococcus aureus, transmission, microtopography, sharklet
Volume I Issue 5 - 2014

\author{
Mann EE,' Mettetal MR,' May RM,' Drinker \\ MC,' Stevenson BC,' Baiamonte VL, ${ }^{3}$ Marso \\ JM, ${ }^{3}$ Dannemiller EA,' Parker AE, ${ }^{4}$ Shravanthi \\ TR,' Sande MK 2,3 \\ 'Sharklet Technologies, Inc., USA \\ ${ }^{2}$ Department of Emergency Medicine, University of Colorado \\ School of Medicine, USA \\ ${ }^{3}$ Work, Education, and Lifelong Learning Simulation Center at \\ University of Colorado Hospital, USA \\ ${ }^{4}$ Center for Biofilm Engineering, and Department of \\ Mathematical Sciences, Montana State University, USA
}

Correspondence: Shravanthi T Reddy, Sharklet Technologies, Inc., I 2635 E Montview Blvd, Suite 155, Aurora, CO 80045, USA, Tel 7208594184,Email sreddy@sharklet.com

Received: November 06, 2014 | Published: December 23,

2014
Abbreviations: HAI, hospital-acquired infections; HCP, healthcare practitioners; CFU, colony forming units; LD, log density; LR, log reduction; LME, linear mixed effect; MP, micro patterned; UNP, Unpatterned; PCA, principal component analysis; AP, attached bacterial load percentages.

\section{Introduction}

Hospital-acquired infections (HAIs) are responsible for clinical complications with an estimated $6.1 \%$ increase in attributable deaths. ${ }^{1,2}$ HAIs increased the length of stay in a hospital from 5.9 to 9.6 days based on one study of over 1,250 patients. ${ }^{1}$ Concomitantly, estimated healthcare costs double for patients with HAIs. ${ }^{1,3}$ As many as $27 \%$ of hospital ward patient rooms are contaminated with $S$. aureus, and as many as $64 \%$ of burn intensive care unit surfaces are contaminated with $S$. aureus. ${ }^{4}$ Cross-contamination of the hands of healthcare practitioners (HCP) previously in direct patient contact or indirectly by touching contaminated environmental surfaces has been shown to contribute to $20-40 \%$ of HAIs. ${ }^{2,5}$ Contaminated hospital surfaces have been carefully investigated in numerous studies and identified to be a major source of HAIs. ${ }^{6-9}$ Despite an improved understanding of the role of environmental contamination, bioburden remains unacceptably high between terminal cleanings. ${ }^{10,11}$ Sampling environmental surfaces following terminal cleanings identified that less than $50 \%$ of the surfaces were truly clean. ${ }^{12-14}$ Microorganisms identified on environmental surfaces include bacteria, viruses, and fungi, each with potential to cause HAIs. Additionally, these microorganisms are capable of surviving desiccation and minimal nutrient environments for extended periods of time, increasing the likelihood of transfer to susceptible patients. ${ }^{8,15,16}$ Contamination of surrounding environmental surfaces has been identified as the most impactful risk of contamination to HCP gloves/hands. ${ }^{17}$ Innovative surface technologies are needed to assist in reducing environmental contamination and preventing infections. Sharklet Technologies, Inc. has developed the Sharklet ${ }^{\mathrm{TM}}$ micropattern to address the need for a novel microorganism-resistant surface. The micropattern is an ordered microtopography engineered to control bioadhesion based on 
basic principles relating to surface energy (Figure 1). ${ }^{18,19}$ Previously, the sharkskin bioinspired micropattern has been shown to inhibit surface contamination, bacterial colonization, and biofilm formation of multiple organisms. ${ }^{20-22}$ Here a simulated clinical scenario was designed to evaluate transfer of bacteria by practicing physician volunteers onto micropatterned surfaces compared to unpatterned surfaces

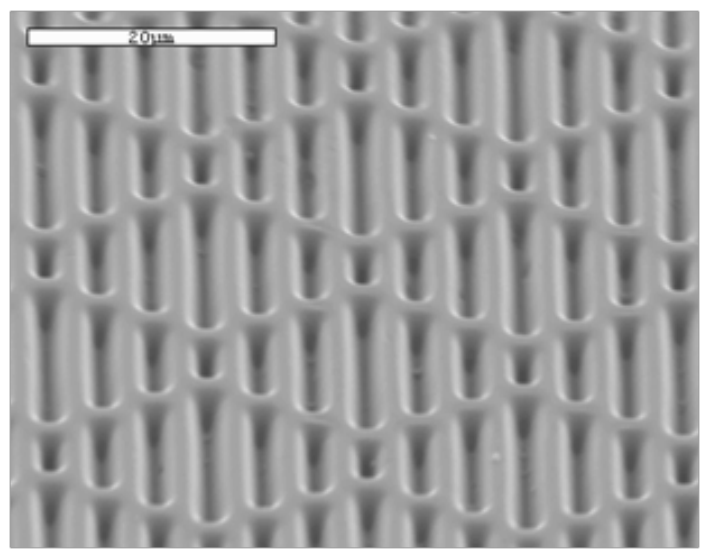

Figure IThe Sharklet micropattern.

Acrylic film embossed with $2 \mu \mathrm{m}$ width/spacing and $3 \mu \mathrm{m}$ deep recessed features in periodic diamond pattern. Measure bar indicates $20 \mu \mathrm{m}$.

\section{Methods}

\section{Materials and bacterial inoculum}

Sharklet micropatterned and unpatterned acrylic film was supplied by Sharklet Technologies, Inc. (Aurora, CO). The micropattern consists of recessed rectangular features arranged in a periodic diamond-shaped pattern (Figure 1). The micropattern feature dimensions are $2 \mu \mathrm{m}$ width/spacing and $3 \mu \mathrm{m}$ depth. Staphylococcus aureus (ATCC 6538) was grown by selecting a single colony from a fresh streak plate and placing it into $100 \mathrm{ml}$ of TSB media overnight on an orbital shaker set to 250 RPM. Bacteria were then diluted to a concentration of $1 \times 10^{8} \mathrm{CFU} / \mathrm{ml}$ in sterile phosphate-buffered saline, using a previously-established standard curve comparing $\mathrm{OD}_{600}$ of overnight culture to $\mathrm{CFU} / \mathrm{ml}$.

\section{Clinical simulation scenario}

Physician volunteers were recruited to participate in a simulated clinical scenario of acute pulmonary embolism in an emergency department setting (Figure 2). IRB approval was obtained for the study (protocol \#13-2931). The study was conducted at the Work, Education, and Lifelong Learning Simulation (WELLS) Center at the University of Colorado Anschutz Medical Center (Aurora, CO). The simulation environment, modeled after an emergency department resuscitation suite, was designed to contain only objects necessary in the clinical care of the patient so as to better control the behaviors and what was touched during the scenario. A high fidelity Sim Man $3 \mathrm{G}$ mannequin (Laerdal, Stavanger, Norway) functioned as the patient in the scenario (Figure 2). Study personnel placed new unpatterned or Sharklet ${ }^{\mathrm{TM}}$ micropatterned films on anticipated touch surfaces prior to the start of each simulation scenario: the handles and along the edges of acode cart, the shock button of a cardiac defibrillator, and an epinephrine medication vial (Figure 2). Fresh control films were placed over femoral pulse pads on the simulation mannequin to provide normalization of total capable bacterial bioburden in each scenario (Figure 2). The final step prior to physician entry into the simulation suite for initiation of the scenario was the inoculation of the left leg of the mannequin circumferentially ankle to knee using a small cotton cloth saturated with approximately $20 \mathrm{ml}$ of bacterial inoculum (Figure 2). The leg was allowed to dry partially for approximately $1 \mathrm{~m}$ before the physician volunteer entered the simulation suite to begin the scenario. The mannequin leg was an appropriate location for inoculation in this scenario testing HCP-mediated transfer of $S$. aureus, a ubiquitous skin colonizer. Examination of the mannequin leg allowed for repeatable inoculation of the HCP gloves, but in clinical situations interaction with any contaminated surface would likely result in contamination of HCP gloves.
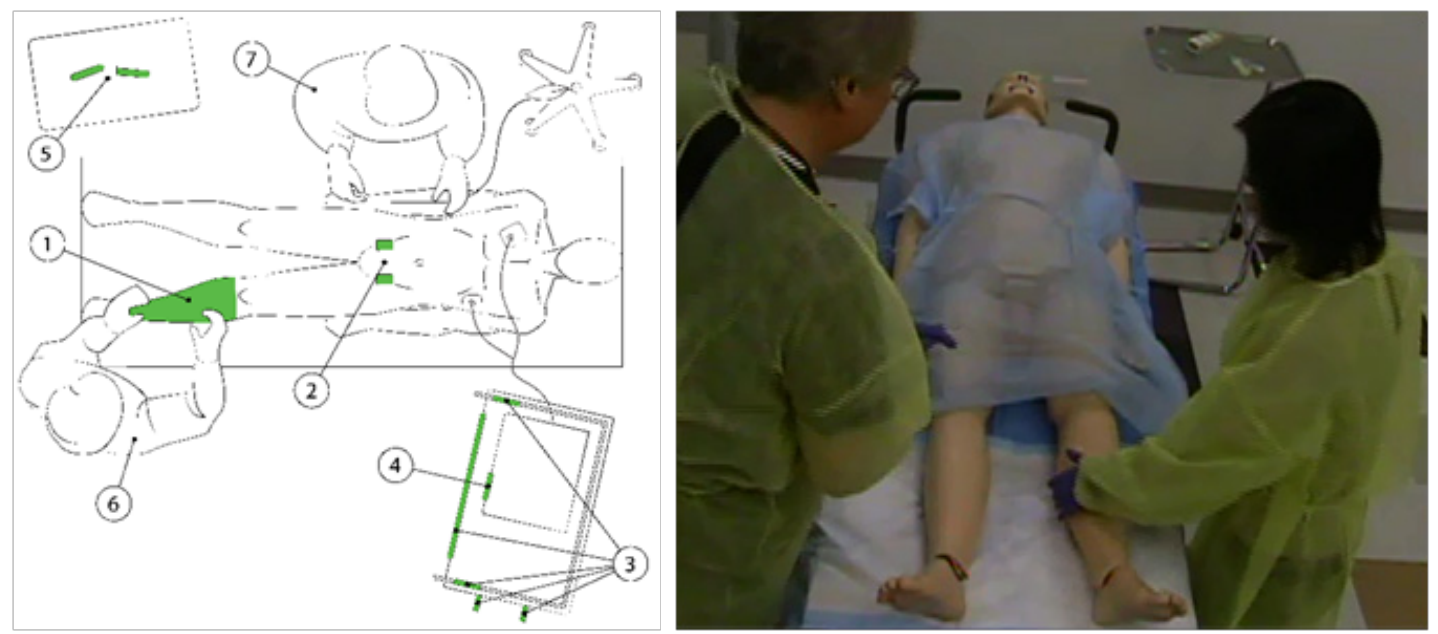

Figure 2 Clinical simulation environment.

A) Simulation room setup included inoculating S. aureus onto the left leg of a simulation mannequin (I). Consenting volunteer physicians (6) blinded to the purpose of the study participated in the clinical scenario along with a supporting nurse (7). Smooth control surfaces were applied over the mannequin's femoral arteries (2) (for normalization of total transfer capability). Either micropatterned or unpatterned surfaces were placed in the procedure room on the code cart (3), cardiac defibrillator shock button (4), and epinephrine 2-part medication vial (5). At the end of the scenario the surfaces were sampled with RODAC plates. B) Image of the actual simulation scenario initiation. 
The simulation suite door was marked as an isolation precaution, and each physician was instructed to don gloves and a gown prior to entry. Upon entry into the simulation resuscitation suite, each physician volunteer encountered a nurse (simulation center actor) who provided the following clinical context (Figure 2): the patient, John Kramer, was a 68 year-old male presenting with complaint of left calf pain and hemoptysis following an $8 \mathrm{~h}$ international flight-highly suggestive of an acute pulmonary embolism. One of two available nurses was randomly assigned to each scenario, and they understood the objectives of the study but were blinded to the type of films used to outfit experimental locations (micropattern or unpatterned). Throughout the scenario, the nurse provided guidance to each physician to ensure the physician completed the anticipated sequence of events successfully as part of the study scenario. The simulated emergency scenario consisted of the following event sequence: following the nurse's providing a clinical synopsis of the patient and his chief complaint of leg pain and hemoptysis, the nurse requested that the physician examine the left calf for tenderness and modeled this behavior using a two-handed technique. The nurse then informed the physician that when bilateral femoral pulses were checked prior to physician entry they seemed unequal. When the physician palpated the femoral pulses on the simulation mannequin, pulses were lost and the cardiac monitor promptly indicated the initiation of cardiac arrest (ventricular fibrillation). The physician had to then move the code cart from its location approximately four feet away from the patient to the patient's bedside while the nurse began providing chest compressions and simulated ventilations. Based upon the underlying cardiac rhythm, the physician initiated Advanced Cardiac Life Support algorithms per the American Heart Association, to include cardiac defibrillation followed by the administration of epinephrine. The physician placed defibrillation pads onto the chest of the simulation mannequin and charged the defibrillator located on top of the code cart. After the physician administered a shock to the mannequin, the nurse directed the physician to an adjacent bedside stand where a two-part vial of epinephrine was available. The physician assembled the two-part medicine vial (glass drug vial and plastic syringe barrel) and subsequently administered the simulated medication into the pre-established intravenous catheter in the antecubital fossa of the mannequin's right arm. Upon administration of this medication, the nurse informed the physician of the conclusion of the scenario. The physician then safely doffed all potentially contaminated personal protective equipment (gown and gloves).

A total of 11 scenarios were randomized so that five scenarios contained unpatterned film and six scenarios contained micropatterned film. Study personnel entered the room immediately after completion of each scenario to remove films, place them on a flat surface, and recover bacteria from the films. The film samples were pressed for $10 \mathrm{~s}$ each with RODAC contact plates (Becton Dickinson, Sparks, MD) to recover any bacteria present. ${ }^{20} \mathrm{RODAC}$ plates were incubated at $37^{\circ} \mathrm{C}$ for $24 \mathrm{hrs}$ and resulting colonies were enumerated as Colony Forming Units (CFU). Multiple RODAC plates were occasionally required for the code cart samples when the cart was handled in multiple areas, and in these cases CFUs were averaged across the plates.

\section{Statistical analysis}

CFUs/RODAC were $\log _{10}$ transformed to $\log$ densities (LD) because, as revealed by normal probability plots, the LDs were approximately normally distributed and analyzed as described below. ${ }^{23,24}$ On RODACs CFU=1 was substituted for zero counts; this occurred six times. When the CFUs were greater than $1000 \mathrm{CFU}$, the total colonies on the plate were quantified to the nearest 100 CFU (occurring a total of 7 times). The highest countable number was $5000 \mathrm{CFU}$. Magnification of high resolution images was used to count dense bacterial colonies when necessary as previously described. ${ }^{20}$ To assess the repeatability of the femoral pulse LDs in each of the micropatterned and unpatterned surface groups, the LDs collected during the femoral pulse check were analyzed separately for each group using a Linear Mixed Effect (LME) model. ${ }^{25}$ The model included nested random effects for physician nested in nurse, and nurse (5-6 physicians were assisted by each of two nurses).

\section{Comparisons between the micropatterned and unpatterned films}

Each LD was normalized to the initial bacterial LD of the femoral pulse, resulting in a $\log$ reduction (LR) value that could then be used to compare the transfer of bacteria onto the micropatterned and unpatterned surfaces. This normalization was an important feature of the design of the scenario due to the expected high variance among physicians interacting with the patient and experimental locations. Normalization allowed for isolation of this variance and a more powerful statistical analysis. Three LRs were calculated for each physician, one LR for each of the 3 test locations (code cart, cardiac defibrillator button, medication vial). Each LR was computed by subtracting the mean LD for the film surface from the mean LD for the femoral pulse pad. For the femoral pulse, code cart, and medication vial surfaces, two LDs were averaged since there was one LD for each hand (right and left). For the cardiac defibrillator, there was only a single LD since the physicians only used right hands (noted for all 11 physicians by independent observers).

The repeatability of the LRs was assessed for each location and group combination separately using LME models with a random effect for the nurse. The LRs for the unpatterned surfaces were more variable among physician volunteers than the LRs for the micropatterned surfaces. To account for this heteroscedastic variance (while ignoring the negligible variance due to nurses) a Welch 2-sample 1-sided $t$ test was used to compare the micropatterned and unpatterned groups for each surface separately. For better interpretability, differences in LRs (Micropatterned (MP) minus Unpatterned (UNP)) are presented as a ratio of attached bacterial load percentages (AP) via the formula $\mathrm{AP}(\mathrm{UNP}) / \mathrm{AP}(\mathrm{MP})=10^{(\mathrm{LR}(\mathrm{MP})-\mathrm{LR}(\mathrm{UNP}))}$. This ratio gives the multiplicative factor describing how many more bacteria are attached to the unpatterned surfaces relative to the micropatterned surfaces. All tests were performed at a significance level of 5\% and implemented in $\mathrm{R}^{26}$ package nlme: Linear and Nonlinear Mixed Effects Models. ${ }^{27}$

\section{Principal component analysis}

Principal component analysis (PCA) was applied to the covariance matrix for the 3 LRs from the code cart, cardiac defibrillator button, and medication vial to assess multi-collinearity (i.e., possible covariance among the LRs from the 3 different locations) and to provide a graphical qualitative description of the LRs. Combined mean LRs for locations with collinearity (defibrillator button and code cart) were compared, again for interpretability, by using a ratio of attached bacterial load percentages (AP) using the equation $\mathrm{AP}(\mathrm{UNP}) / \mathrm{AP}(\mathrm{MP})=10^{(\mathrm{LR}(\mathrm{UNP})-\mathrm{LR}(\mathrm{MP}))}$.

\section{Results}

The log reduction (LR) for each test location represented the reduced load of organisms carried from the first control location 
touched to ensuing locations (Figure 3). The geometric mean CFU/ RODAC at the first control location touched at the femoral pulse pads (Figure 2) ranged from 175-250 CFU/RODAC (95\% confidence interval). This allowed for comparison of the transmission reduction for the micropattern and the unpatterned surfaces. The micropatterned surface exhibited a mean LR for the cardiac defibrillator $(\mathrm{LR}=2.26)$ that was significantly larger than the unpatterned surface $(\mathrm{LR}=.12$, $p=0.023$ ) (Table 1). The micropattern had larger mean LRs on the code cart $(\mathrm{LR}=1.29)$ and medication vial $(\mathrm{LR}=1.88)$ compared to the unpatterned surface LRs on the same locations $(\mathrm{LR}=0.48)$ and $(\mathrm{LR}=1.30)$, respectively (Table 1). Therefore, the differences in LRs ranged from 0.58 to 1.14 larger on the micropattern compared to the unpatterned surfaces.

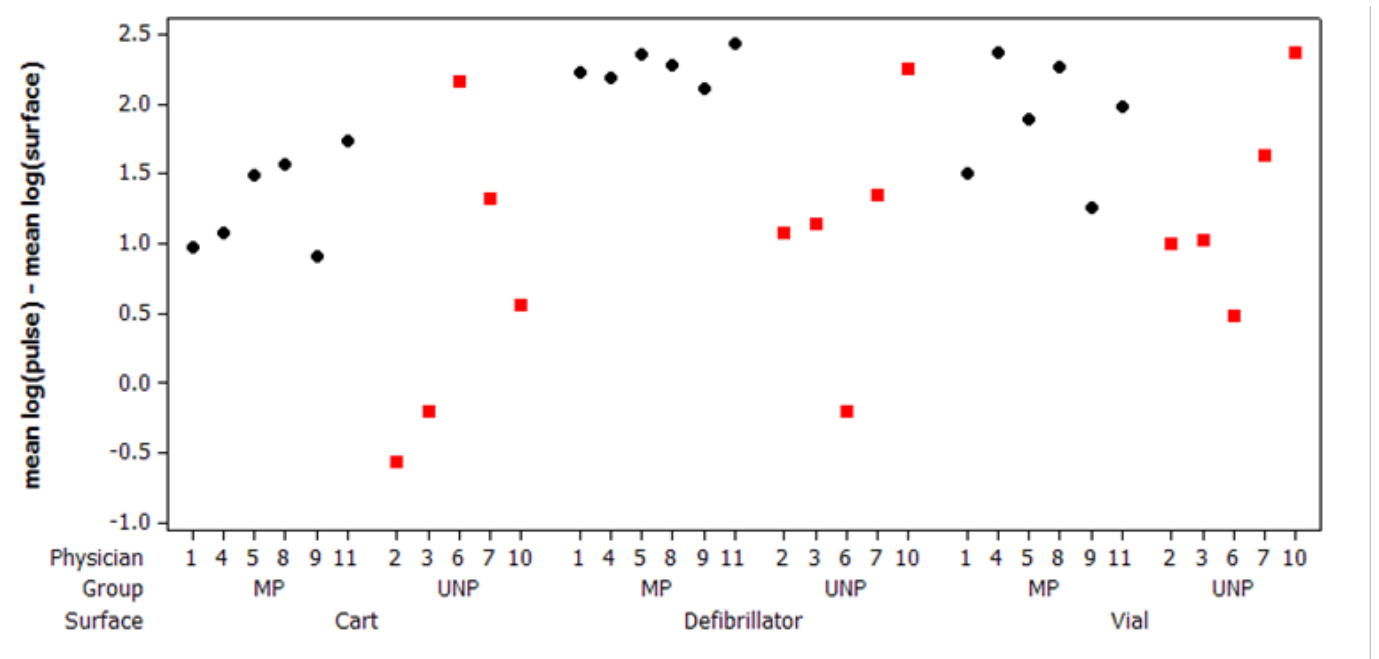

Figure 3 Individual bacterial log reduction values on experimental surfaces with respect to the control surface.

Each simulation scenario resulted in a set of unpatterned (red squares) or micropatterned (black diamond's) bacterial log densities for each location which were subtracted from the initial pulse bacterial density within the same scenario. (MP, micropatterned; LR, log reduction).

Table I Comparison of the mean log reduction values with respect to the control surface load

\begin{tabular}{|c|c|c|c|c|c|c|c|c|c|}
\hline \multirow{2}{*}{ Location } & \multirow{2}{*}{ Group } & \multirow{2}{*}{ PR\% } & \multirow{2}{*}{$\begin{array}{l}\text { Mean } \\
\text { LR }\end{array}$} & \multicolumn{2}{|l|}{ Variance } & \multirow{2}{*}{ SD } & \multirow{2}{*}{$\begin{array}{l}\text { mean } \\
\text { LR } \Delta\end{array}$} & \multirow{2}{*}{$\begin{array}{l}\text { Relative } \\
\text { transmission } \\
\text { reduction }\end{array}$} & \multirow{2}{*}{$p$} \\
\hline & & & & Among-physician & Among-nurse & & & & \\
\hline \multirow[b]{2}{*}{ Cart } & $M P$ & 95 & 1.29 & $0.119(100 \%)$ & $0(0 \%)$ & 0.345 & \multirow[b]{2}{*}{0.64} & \multirow[b]{2}{*}{$4.4 x$} & \multirow[b]{2}{*}{0.146} \\
\hline & UNP & 78 & 0.66 & I.24 (I00\%) & $0(0 \%)$ & 1.12 & & & \\
\hline \multirow[b]{2}{*}{ Defibrillator } & $M P$ & 99.5 & 2.26 & $0.014(100 \%)$ & $0(0 \%)$ & 0.119 & \multirow[b]{2}{*}{$\mathrm{I} .14$} & \multirow[b]{2}{*}{$13.8 x$} & \multirow[b]{2}{*}{0.023} \\
\hline & UNP & 92 & 1.12 & $0.774(100 \%)$ & $0(0 \%)$ & 0.88 & & & \\
\hline \multirow{2}{*}{ Vial } & MP & 99 & 1.88 & $0.1855(100 \%)$ & $0(0 \%)$ & 0.431 & \multirow{2}{*}{0.58} & \multirow{2}{*}{$3.8 x$} & \multirow{2}{*}{0.083} \\
\hline & UNP & 95 & 1.3 & $0.5188(100 \%)$ & $0(0 \%)$ & 0.72 & & & \\
\hline
\end{tabular}

Bacterial log densities were normalized to the mannequin femoral pulse control surfaces for the unpatterned (UNP) or micropatterned (MP) surfaces within a simulation scenario. The resulting log reduction (LR) values from the control femoral pulse surface to the experimental unpatterned or micropatterned surface were directly compared to evaluate efficiency of bacterial transmission onto micropatterned surfaces. The reported repeatability standard deviation (SD) is the square root of the sum of the among-physician and among-nurse variances. PR (\%) refers to the percent reduction calculated by I-I0^(-LR).

The $S$. aureus LDs recovered from the control femoral pulse pad location in the 2 groups exhibited a mean $\mathrm{LD} \pm$ (repeatability SD) of $2.53 \pm 0.40$ for the micropatterned group and $2.21 \pm 0.826$ for the unpatterned control group. To ascertain where variability existed in the experiment, the among-nurse, among-physician, and withinphysician variances were compared. $100 \%$ of the variability was attributable to the within-physician variance. Each point in the PCA biplot in (Figure 4) is a single representation of the LRs associated with the 3 experimental locations for each physician volunteer. The biplot suggests a partitioning of the LRs with respect to pattern type. The fact that the micropattern points congregate to the right of the 3 vectors provides a qualitative picture that the micropattern LRs are typically larger than the LRs for the unpatterned group. The magnitude of the PCA loadings for the code cart and cardiac defibrillator button were similar but distinct from the medication vial (i.e., the vector for the vial LRs in the biplot is shorter and flatter than the other vectors). Therefore it is appropriate to combine the LRs for the code cart and cardiac defibrillator to compare bacterial transfer between the micropattern and unpatterned surfaces (Table 2). In doing so the mean $\mathrm{LR} \pm \mathrm{SD}$ of the micropatterned surface with respect to the femoral pulse location was $1.81 \pm 0.326$ compared to $1.08 \pm 0.802$ for the unpatterned surface with a difference of $0.73(p=0.058)$. This result shows that 5.4 times more bacteria (accounting for the logarithmic transformation) attached to the unpatterned surfaces, when averaging across the cart and defibrillator surfaces, compared to the micropatterned surfaces. 
Table 2 Analysis of mean log reduction for the cart and defibrillator surfaces with respect to the femoral pulse bacterial load

\begin{tabular}{llllll}
\hline Group & mean LR & SD & Mean Difference & Relative transmission reduction & $\boldsymbol{p}$ \\
\hline Micropatterned & $\mathrm{I} .81$ & 0.326 & 0.73 & $5.4 \times$ & 0.058 \\
Unpatterned & $\mathrm{I} .08$ & 0.802 & &
\end{tabular}

Unpatterned or micropatterned log reductions relative to the femoral pulse log densities were pooled and compared. (LR, log reduction; SD, standard deviation).

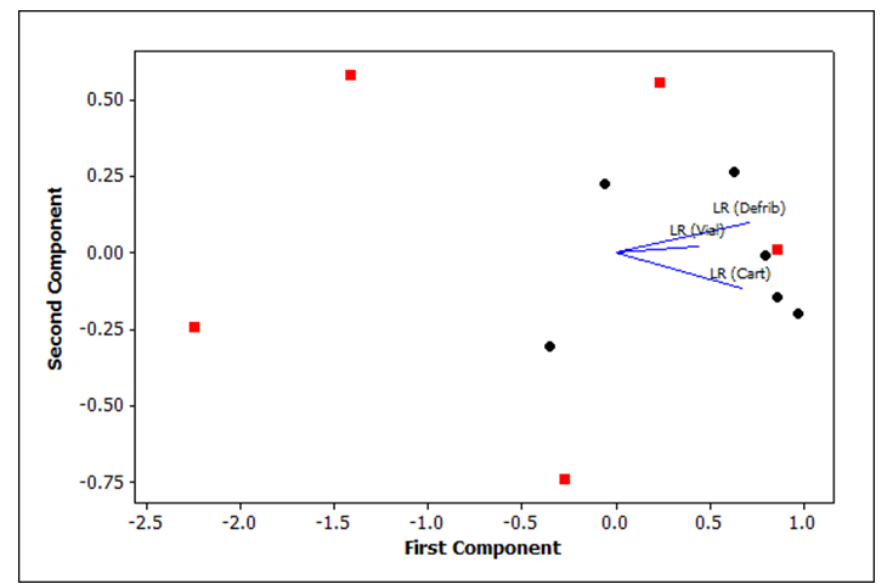

Figure 4 Principle component analysis indicates the potential for the micropattern reduction.

Black circles indicate measurements from physician volunteers in the micropatterned surface group; red squares indicate measurements from physician volunteers in the unpatterned surface group. (PCA, principle component analysis; LR,log reduction).

\section{Discussion}

This study was designed to evaluate the ability of Sharklet ${ }^{\mathrm{TM}}$ micropatterned surfaces to reduce bacterial transfer compared to unpatterned surfaces when tested within a simulated emergency department clinical environment. Unlike previous studies examining bacterial transfer and persistence in a laboratory setting, ${ }^{20}$ a simulated hospital setting involving practicing physician volunteers was chosen so as to closely mimic hospital conditions. The medical equipment included in this study and the interactions between clinicians and this equipment are the same as would be exhibited in a clinical environment where patients are being treated. Participating clinicians were blinded to the study objectives such that they interacted with the environment and equipment as they would in their typical practice. While tracking environmental bacteria located on actual hospital surfaces would offer the most realistic approach, the simulation center provided a safe and efficient way to confirm laboratory results recently published on the micropattern technology. ${ }^{20}$

Bacterial contamination testing, even in a simulation environment, requires highly controlled and established methods to track bacterial adherence to surfaces while avoiding inherent variability involved with human studies (in this case, volunteer physicians). Previous laboratory experiments were the foundation for simulation scenario testing. Bacterial suspensions used $\left(1 \times 10^{8} \mathrm{CFU} / \mathrm{ml}\right)$ in the simulation scenarios were concentrated to consistently observe transfer of bacteria among multiple touches throughout the designed scenario. Experimental testing and surface sampling methods were developed previously ${ }^{20}$ based on evidence that the micro pattern's enhanced surface energy ${ }^{18,19}$ is more resistant to microorganism adherence compared to unpatterned surfaces. ${ }^{20-22,28}$ Bacteria transfer was repeatably reduced where the micropattern was present on common objects in the hospital simulation room compared to unpatterned surfaces (Tables $1 \& 2$ ) (Figure 3). The micropatterned surface reduced bacterial transmission by more than one $\log$ on the defibrillator button location compared to the unpatterned surface $(p=0.023)$. Put another way, more than 13 times more bacteria were transferred to the defibrillator button when an unpatterned surface was present compared to a micropatterned surface (Table 1). On the code cart and medication vial 4.4 and 3.8 times more bacteria were transmitted to the unpatterned surface respectively compared to the micropatterned surface (Table 1). Although the code cart and medication vial results were not statistically significant at a significance level of 5\% (i.e., the associated $p$-values of 0.146 and 0.086 were both larger than 0.05 ); they are suggestive that a larger study with more participating physicians will provide statistically significant results.

Importantly, each scenario included a normalization step by sampling transfer to control surfaces on the mannequin's femoral pulse that allowed for comparisons across multiple physicians (Table 1). Analysis of the log densities on the femoral pulse indicates that bacterial transfer was repeatable regardless of surface type present in the scenario. The statistical modeling estimated that the different nurses did not contribute to the variability of the LRs, leaving physicians to be the sole source of variance in the quantitative bacterial LR (Table 1). Nonetheless, the physician-to-physician variance was sufficiently minimal to produce significant results with as few as 11 physician volunteers. This indicates strong repeatability among the 11 physician volunteers and 2 nurses that participated in the study, as well as efficient sampling methods and laboratory techniques.

The linear relationships of the LRs among the locations were evaluated using principal component analysis (PCA). PCA also provides a qualitative graphical summary of the LRs across the 3 locations (Figure 4). The code cart micropattern LR exhibited transmission that covaried with the defibrillator button (Table 1). The medication vial with micropatterned surface reduced transmission, but the LR covaried much less with either of the other two locations. Therefore, the code cart and defibrillator button were combined to obtain an average bacterial transmission reduction among covarying locations. Over 5 times more bacteria were transmitted onto the unpatterned surfaces compared to the micropatterned surfaces at the code cart and defibrillator button locations combined (Table 2).

\section{Conclusion}

Bacterial transmission was reduced by a factor of 5.4 on the micropatterned surface compared to the corresponding unpatterned surface for the two locations that exhibited covariance. Importantly, reduced transmission occurred at all locations tested in this study but maximally at the defibrillator button by 13.8 times. Given that unpatterned surfaces allow more bacterial transmission, contributing to the spread of S. aureus on surfaces, there could be major benefits to 
patient safety in implementing this micropatterned surface that combats bacterial spread. S. aureus was used in this study as a representative and common bacterial species, but the micropattern has been shown to reduce contamination of a wide range of microbial species in other studies. ${ }^{21,22}$ It is reasonable to expect the micropatterned surface to broadly reduce microbial transmission occurring on hospital surfaces. The Sharklet micropattern is a valuable option to combat transient surface contamination on high touch surfaces, especially in hospitals and healthcare settings. This study demonstrates the ability of the micropattern to offer reduced transmission of $S$. aureus on hospital high-touch surfaces in simulated physician-patient-environment interaction scenarios.

\section{Acknowledgments}

None.

\section{Conflicts of interest}

EEM, MRM, RMM, MCD, BCS, EAD, and STR are paid employees of Sharklet Technologies, Inc. AEP is a paid consultant of Sharklet Technologies, Inc.

\section{References}

1. Roberts RR, Scott RD, Hota B, et al. Costs attributable to healthcareacquired infection in hospitalized adults and a comparison of economic methods. Med Care. 2010;48(11):1026-1035.

2. Weber DJ, Rutala WA, Miller MB, et al. Role of hospital surfaces in the transmission of emerging health care-associated pathogens: norovirus, Clostridium difficile, and Acinetobacter species. Am J Infect Control. 2010;38(5 Suppl 1):S25-33.

3. Roberts RR, Scott RD, Cordell R, et al. The use of economic modeling to determine the hospital costs associated with nosocomial infections. Clin Infect Dis. 2003;36(11):1424-1432.

4. Boyce JM. Environmental contamination makes an important contribution to hospital infection. J Hosp Infect. 2007;65(Suppl 2):50-54.

5. Falagas ME, Thomaidis PC, Kotsantis IK, et al. Airborne hydrogen peroxide for disinfection of the hospital environment and infection control: a systematic review. J Hosp Infect. 2011;78(3):171-177.

6. Boyce JM, Potter-Bynoe G, Chenevert C, et al. Environmental contamination due to methicillin-resistant Staphylococcus aureus: possible infection control implications. Infect Control Hosp Epidemiol. 1997;18(9):622-627.

7. Carling PC, Huang SS. Improving healthcare environmental cleaning and disinfection: current and evolving issues. Infect Control Hosp Epidemiol. 2013;34(5):507-513.

8. Otter JA, Yezli S, Salkeld JA, et al. Evidence that contaminated surfaces contribute to the transmission of hospital pathogens and an overview of strategies to address contaminated surfaces in hospital settings. Am J Infect Control. 2013;41(5 Suppl):S6-S11.

9. Trick WE, Weinstein RA, DeMarais PL, et al. Comparison of routine glove use and contact-isolation precautions to prevent transmission of multidrug-resistant bacteria in a long-term care facility. $J$ Am Geriatr Soc. 2004;52(12):2003-2009.

10. Weber DJ, Anderson D, Rutala WA. The role of the surface environment in healthcare-associated infections. Curr Opin Infect Dis. 2013;26(4):338-344.
11. Weber DJ, Rutala WA. Self-disinfecting surfaces: review of current methodologies and future prospects. Am J Infect Control. 2013;41(5 Suppl):S31-S35.

12. Carling PC, Parry MF, Bruno-Murtha LA, et al. Improving environmental hygiene in 27 intensive care units to decrease multidrug-resistant bacterial transmission. Crit Care Med. 2010;38(4):1054-1059.

13. Carling PC, Parry MF, Von Beheren SM, et al. Identifying opportunities to enhance environmental cleaning in 23 acute care hospitals. Infect Control Hosp Epidemiol. 2008;29(1):1-7.

14. Havill NL. Best practices in disinfection of noncritical surfaces in the health care setting: creating a bundle for success. Am J Infect Control. 2013;41(5 Suppl):S26-30.

15. Abreu AC, Tavares RR, Borges A, et al. Current and emergent strategies for disinfection of hospital environments. J Antimicrob Chemother. 2013;68(12):2718-2732.

16. Huang SS, Datta R, Platt R. Risk of acquiring antibiotic-resistant bacteria from prior room occupants. Arch Intern Med. 2006;166(18):1945-1951.

17. Morgan DJ, Rogawski E, Thom KA, et al. Transfer of multidrugresistant bacteria to healthcare workers' gloves and gowns after patient contact increases with environmental contamination. Crit Care Med. 2012;40(4):1045-1051.

18. Decker JT, Kirschner CM, Long CJ, et al. Engineered antifouling microtopographies: an energetic model that predicts cell attachment. Langmuir. 2013;29(42):13023-13030.

19. Long CJ, Finlay JA, Callow ME, et al. Engineered antifouling microtopographies: mapping preferential and inhibitory microenvironments for zoospore attachment. Biofouling. 2010;26(8):941-952.

20. Mann EE, Manna D, Mettetal MR, et al. Surface micropattern limits bacterial contamination. Antimicrob Resist Infect Control. 2014;3:28.

21. May RM, Hoffman MG, Sogo MJ, et al. Micro-patterned surfaces reduce bacterial colonization and biofilm formation in vitro: Potential for enhancing endotracheal tube designs. Clin Transl Med. 2014;3:8.

22. Reddy ST, Chung KK, McDaniel CJ, et al. Micropatterned surfaces for reducing the risk of catheter-associated urinary tract infection: an in vitro study on the effect of sharklet micropatterned surfaces to inhibit bacterial colonization and migration of uropathogenic Escherichia coli. $J$ Endourol. 2011;25(9):1547-1552.

23. Hamilton MA, Hamilton GC, Goeres DM, et al. Guidelines for the statistical analysis of a collaborative study of a laboratory method for testing disinfectant product performance. J AOAC Int. 2013;96(5):1138-1151.

24. Jarvis B. Statistical Aspects of the Microbiological Examination of Foods. 2nd ed. Elsevier: San Diego, Calfonia, USA; 2008.

25. Pinheiro JC, Bates D. Mixed-Effects Models in S and S-PLUS. Statistics and Computing Series; 2000.

26. Pinheiro J, Bates DM, DebRoy S, et al. R: A language and environment for statistical computing. R Foundation for Statistical Computing; 2013.

27. Pinheiro J, Bates D, DebRoy S, et al. nlme: Linear and Nonlinear Mixed Effects Models. (R package version 3.1-111); 2014.

28. Chung KK, Schumacher JF, Sampson EM, et al. Impact of engineered surface microtopography on biofilm formation of Staphylococcus aureus. Biointerphases. 2007;2(2):89-94. 